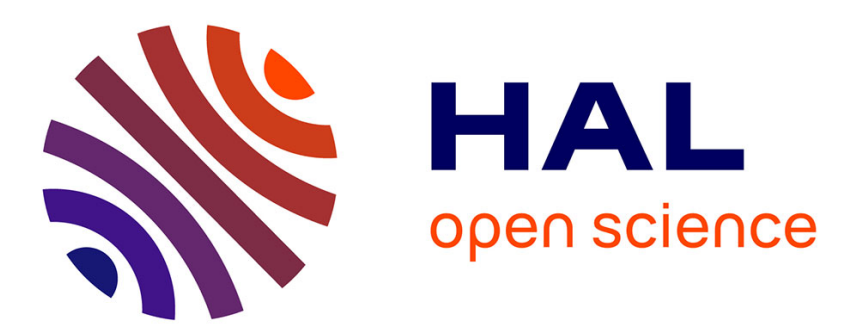

\title{
Le " divin " Platon à la table des Grecs et des Romains : Dynamiques et enjeux de la fabrique d'une mémoire savante dans l'Empire gréco-romain
}

Anthony Andurand, Corinne Bonnet

\section{- To cite this version: \\ Anthony Andurand, Corinne Bonnet. Le " divin " Platon à la table des Grecs et des Romains : Dynamiques et enjeux de la fabrique d'une mémoire savante dans l'Empire gréco-romain. Figures mythiques et discours religieux dans l'Empire gréco-romain, 2019, 10.1484/M.RRR-EB.5.115808 . hal-02288285}

\section{HAL Id: hal-02288285 \\ https://hal.science/hal-02288285}

Submitted on 9 Nov 2019

HAL is a multi-disciplinary open access archive for the deposit and dissemination of scientific research documents, whether they are published or not. The documents may come from teaching and research institutions in France or abroad, or from public or private research centers.
L'archive ouverte pluridisciplinaire HAL, est destinée au dépôt et à la diffusion de documents scientifiques de niveau recherche, publiés ou non, émanant des établissements d'enseignement et de recherche français ou étrangers, des laboratoires publics ou privés. 


\section{Le « divin » Platon à la table des Grecs et des Romains: dynamiques et enjeux de la fabrique d'une mémoire savante dans l'Empire gréco-romain}

\section{Introduction}

La littérature de banquet offre un matériau approprié pour cerner, sur le terrain des cercles de lettrés et des communautés savantes, les dynamiques qui accompagnent et favorisent l'épanouissement d'un Empire «gréco-romain », cette unité que Paul Veyne a définie comme un «fait de culture grecque et de pouvoir romain ${ }^{1} \gg$. De Plutarque à Athénée, le banquet se présente en effet comme un espace stratégique de débats, de partage et de médiation, où se dessinent les contours et la mémoire d'une « cité des savants » à vocation universelle, nourrie de savoirs et de connivence. Appréhendé à l'intersection des communautés, des lieux, des pratiques et des textes qu'il met en relation, le microcosme du banquet offre ainsi un riche observatoire pour ressaisir les codes et les ramifications d'une République «gréco-romaine » des lettres, modelée par le référent culturel de l'hellénisme et attirée par les réseaux du pouvoir romain. C'est à la lumière de ces perspectives que nous nous proposons d'envisager le traitement des figures exemplaires du passé gréco-romain dans les Propos de table, à travers une figure à la fois singulière et évocatrice : celle du « divin Platon $^{2} \gg(\theta \varepsilon \tilde{\varepsilon} о \varsigma \Pi \lambda \dot{\alpha} \tau \omega \nu)$

Rédigée dans les premières années du $\mathrm{II}^{\mathrm{e}}$ siècle de notre ère, cette œuvre se présente comme un recueil de récits de souvenirs, composé à la demande d'un ami romain de Plutarque, Sosius Sénécion. Puisant directement leur inspiration et leur matière dans l'activité intellectuelle et l'expérience sociale du philosophe, les Propos de table restituent le « petit monde » de Plutarque, cette élégante et savante compagnie

P. Veyne, L'Empire gréco-romain, Paris, 2005, p. 10.

2 Plutarque, Périclès, 8, 2: «Car l'élévation d'esprit et l'efficacité si parfaite dans l'exécution qui, pour reprendre le mot du divin Platon $\left(\theta \varepsilon \tilde{c} \varsigma_{\zeta} \Pi \lambda \alpha \dot{\tau} \tau \omega v\right)$, "s'ajoutèrent à ses dons naturels", il [= Périclès] les dut à "la science physique à laquelle il emprunta, pour son éloquence, tout ce qui pouvait lui convenir". Ce fut ainsi qu'il surpassa, de loin, tous les autres orateurs » (trad. A.-M. Ozanam, Paris, 2001).

Anthony Andurand Université Toulouse - Jean Jaurès

Corinne Bonnet Université Toulouse - Jean Jaurès

Figures mythiques et discours religieux dans l'Empire gréco-romain, Textes réunis et édités par Frédéric Chapot, Johann Goeken et Maud Pfaff-Reydellet, Turnhout, Brepols 2018 (p. 17-31) 
réunissant des familiers et des connaissances du philosophe, des philosophes et des lettrés, des notables locaux et de hauts dignitaires de l'administration impériale, venus de diverses régions de l'Empire. L'œuvre met en scène les « conversations

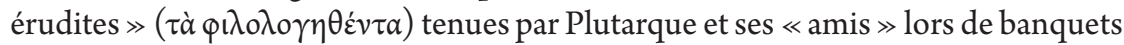
donnés « tant chez vous autres à Rome que chez nous en Grèce (

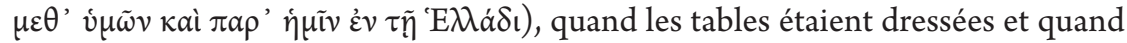
les coupes circulaient ${ }^{3} \gg$. Comme exercice de ressouvenir, empruntant ses codes aux pratiques et aux traditions littéraires liées à la commensalité gréco-romaine, la narration proposée construit les Propos de table comme une riche polyphonie savante, rythmée par les «questions » $(\pi \rho \circ \beta \lambda \eta \dot{\eta} \mu \tau \alpha)$ successivement soumises à l'expertise des convives.

C'est au registre mémoriel que le narrateur, selon le programme esquissé dès le prologue du premier livre, entend rattacher l'évocation de ces «souvenirs » sympotiques. Méditant les vertus respectives du souvenir et de l'oubli dans la sphère du banquet, Plutarque adresse à Sosius Sénécion les paroles suivantes ${ }^{4}$ :

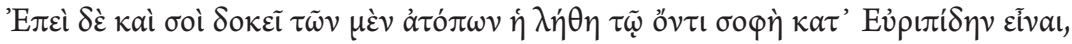

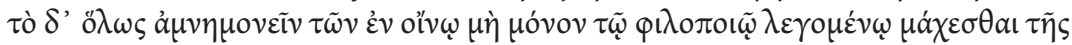

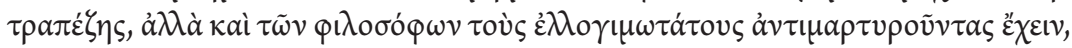

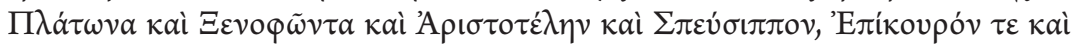

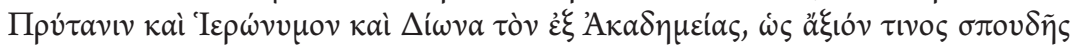

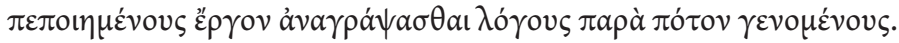

Quant à toi, tu considères, de même, que l'oubli des inconvenances est sagesse en vérité, selon le mot d'Euripide, mais aussi que de perdre complètement

3 Plutarque, Propos de table, I (prologue), 612 E (trad. F. Fuhrmann [livres I-VI], CUF, Paris, 19721978 ; F. Frazier, J. Sirinelli [livres VII-IX], CUF, Paris, 1996). Les neuf livres des Propos de table font ainsi intervenir une petite centaine de personnages d'origines géographiques diverses et de domaines de spécialisation variés, identifiables au moins par un nom (sur la prosopographie des cercles de la sociabilité plutarquéenne, on consultera l'excellente synthèse de B. PUECH, « Les amis de Plutarque », dans Aufstieg und Niedergang der römischen Welt, II, 33, 6, Berlin / New York, 1992, p. 48314893). Les banquets évoqués dans les « souvenirs » sympotiques de Plutarque sont, eux, au nombre d'une soixantaine et localisés, dans leur immense majorité, dans les cités grecques d'Achaïe, en particulier dans la région qui s'étend d'Athènes aux Thermopyles. Le seul banquet situé hors de Grèce

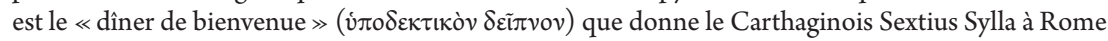
(VIII, 7-8), à l'occasion d'une visite de Plutarque dans l'Urbs. Dans cette perspective, le croisement des données liées au parcours des personnages et à la localisation des réunions offre la possibilité de restituer les logiques relationnelles et géographiques qui structurent le monde plutarquéen des banquets des lettrés et la communauté intellectuelle qu'il met en scène, à la fois enracinée dans le microcosme des cités d'Achaïe et ouverte sur l'Empire. Sur ces aspects, voir les propositions que nous avons formulées récemment au moyen des outils de l'analyse de réseaux : A. ANDURAND, « Le monde plutarquéen des banquets savants : essai dapproche spatiale », Histoire et informatique, 18, 2015, p. 46-53 ; A. Andurand, C. Bonnet, « "Les coutumes et les lois des nations barbares" (Plut., QC 2, 1). Réseaux savants entre centre et périphériedans les Propos de table de Plutarque », dans S. Aufrère, F. Möri (éd.), Les sagesses barbares. Échanges et réappropriations dans l'espace culturel gréco-romain, Neuchâtel, 2015, p. 109-141.

4 Plutarque, Propos de table, I (prologue), 612 D-E. 
le souvenir de ce qui s'est passé à table est contraire à l'opinion commune selon laquelle la table fait les amis, et, de plus, s'oppose au témoignage des plus illustres philosophes: Platon, Xénophon, Aristote, Speusippe, Épicure, Prytanis, Hiéronymos et Dion l'Académicien, qui ont regardé comme une œuvre digne de quelque intérêt de consigner par écrit des propos tenus dans des banquets.

Placé au seuil du recueil, ce passage explicite les traditions auxquelles on peut rattacher le projet d'écriture plutarquéen. En se proposant de consigner par écrit,

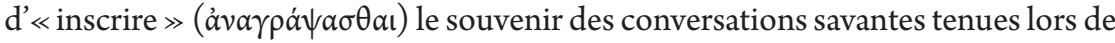
banquets, Plutarque entend faire une œuvre de mémoire, composée sur le mode de l'ảvá, du rappel et de la réactivation : anamnèse de réunions placées sous le signe de la $\varphi \backslash \lambda$ ía et de la connivence érudite, les Propos de table sont aussi et indissociablement, comme le suggère l'énumération des philosophes qui ont précédé l'auteur dans cet exercice, une anabase, une remontrée aux sources de la mémoire et des traditions culturelles de l'hellénisme, dans la conversation engagée, au présent, avec les figures et les œuvres du savoir grec.

Ces variations sur le thème de la mémoire constituent une voie possible pour éclairer le traitement de la figure platonicienne dans les Propos de table. Dans les prologues adressés à Sosius Sénécion comme dans la succession des séquences narratives qui composent chaque livre, le philosophe se présente en effet comme l'une des références privilégiées de l'univers plutarquéen des banquets de lettrés. À travers l'analyse de cette figure tutélaire et de la signification dont elle est investie dans les Propos de table, il s'agit dès lors de mettre en lumière les opérations et les mécanismes que mobilise, dans l'espace d'une République «gréco-romaine » des lettres, la fabrique d'une mémoire savante partagée, ancrée dans une figure mythique, ou «mythifiée ». Attentive, dans un premier temps, aux marqueurs de la présence platonicienne dans plusieurs des aspects offerts par le texte des Propos de table, la démarche visera ensuite à interroger la fonction symbolique que revêt la figure de Platon dans le recueil, comme «saint patron » de la communauté philosophique plutarquéenne et parangon d'un hellénisme désormais élargi aux dimensions de la pax romana. À travers l'éclairage de la figure platonicienne, l'analyse se donnera en outre comme objectif d'explorer l'entrelacement de la « mémoire » et de la « présence » des traditions savantes grecques à partir desquelles se construit, au croisement de l'héritage de l'hellénisme et des données nouvelles de l'ordre romain, le modèle plutarquéen du banquet comme microcosme de l'Empire.

\section{Présences de Platon dans les Propos de table}

La présence de Platon se signale à chacun des niveaux du texte plutarquéen, où la figure du philosophe est investie, par touches successives, d'une signification continuellement enrichie. 
Le nom de Platon est d'abord évoqué dans trois des neuf prologues 5 , qui consistent, pour reprendre la distinction ${ }^{6}$ établie au début du livre II, non en des propos de table

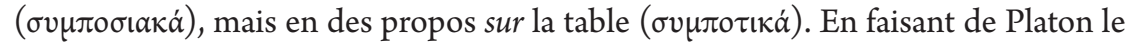

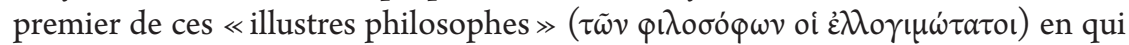
Plutarque reconnaît ses prédécesseurs et en établissant une filiation directe avec l'archétype du Banquet, la référence platonicienne, dès le prologue du premier livre, tend ainsi à inscrire les Propos de table dans la continuité d'une tradition littéraire et philosophique - dont Plutarque, en l'adaptant aux données nouvelles de la période impériale, devait du reste profondément renouveler les codes et le genre.

Le contenu des séquences narratives et des questions réunies dans le recueil témoigne, d'une manière similaire, de la place de Platon dans le modèle sympotique plutarquéen. Sur ce point, les conversations rapportées et mises en scène dans les Propos de table, réglées par des pratiques codifiées et ritualisées 7 , semblent se dérouler selon un scénario homogène. Une fois le sujet arrêté et la question formulée, les symposiastes sont appelés, à tour de rôle, à apporter leur contribution $\left(\sigma v \mu \beta a ́ \lambda \lambda_{\varepsilon \sigma \theta \alpha \iota)}\right.$ aux recherches

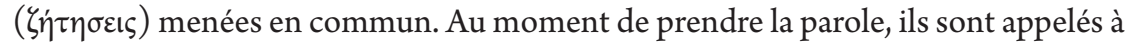

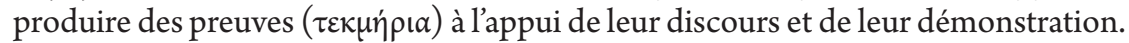
Ces preuves peuvent être déduites de l'expérience ou de l'observation quotidienne. Dans la plupart des cas, cependant, elles sappuient sur le témoignage ( $\mu$ a $\tau$ v́pıov) de ceux que les participants désignent comme les « Anciens ${ }^{8} \gg$ (oi $\pi \alpha \lambda \alpha$ เoi) : il peut s'agir d'une citation, d'un argument, d'une théorie, d'une image ou d'une anecdote, emprunté à tel auteur ou à telle œuvre du passé grec ${ }^{9}$.

Dans chacun des aspects de ce jeu érudit, conçu comme exploration et performance collectives des savoirs, mobilisant l'ensemble des registres de la connaissance et de la mémoire culturelle grecque, Platon occupe une place tout à fait singulière. Les recherches menées à la table de Plutarque témoignent assurément d'une grande familiarité avec l'œuvre du philosophe, largement commune aux Grecs et aux Romains. Platon apparaît d'abord comme l'un des sujets de prédilection des convives : quatre

5 La référence au Banquet platonicien intervient dans le prologue du livre I ( $612 \mathrm{E}$ ) et dans celui du livre VI (686 A-D), au début duquel Plutarque évoque par ailleurs la simplicité, «tout à fait digne

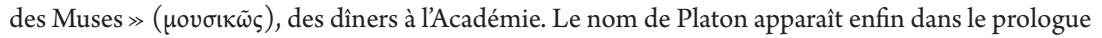
du livre III (645 A), qui emprunte aux Lois (I, 649 d-650 a) l'idée selon laquelle le vin est le meilleur

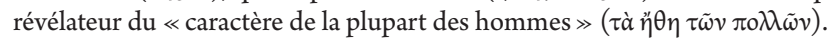

6 Plutarque, Propos de table, II (prologue), 629D.

7 Sur les codes et les règles qui sous-tendent le partage de la parole et des savoirs dans l'espace sympotique mis en scène dans les Propos de table: J. KöNIG, Saints and Symposiasts. The Literature of Food and the Symposium in Greco-Roman and Early Christian Culture, Oxford, 2012, p. 66-81.

8 Sur les vertus attachées aux Anciens dans l'œeture de Plutarque, voir C. BRÉCHET, « Les palaioi chez Plutarque », dans B. BAKHouche (éd.), L'ancienneté chez les Anciens, Montpellier, 2 vol., 2003, II, p. 519-550. On rappellera ici la conclusion de l’article (p. 550) : «L'œuvre de Plutarque témoigne ainsi de la participation dynamique des palaioi ane pensée, dans un foisonnement de vie dont les discussions des Propos de table sont sans doute la preuve la plus éloquente ».

9 Du passé grec seulement : l'ensemble des références intellectuelles convoquées par les convives de Plutarque se rattachent, sans exception, aux traditions de l'hellénisme. 
des questions ${ }^{10}$ réunies dans le recueil s'apparentent pour ainsi dire au genre des «questions platoniciennes », auquel Plutarque s'est également essayé, et puisent directement leur source - le cas est plutôt rare dans les Propos de table ${ }^{11}$ - dans la fréquentation et l'étude des dialogues de Platon. Le philosophe, par ailleurs, occupe une place de choix parmi les témoins et les autorités mobilisés, discutés ou cités au cours des conversations, derrière Homère, certes, mais devant tous les autres auteurs du passé grec ${ }^{12}$. Les dialogues les plus fréquemment soumis à la discussion, dans cette perspective, sont, dans l'ordre, le Banquet, la République et le Timée, le Phèdre et les Lois.

La tonalité platonicienne des discussions, envisagées du point de vue des thèmes abordés, des matériaux de recherche ou des références mobilisés, s'accorde avec la composition intellectuelle du groupe mis en scène dans les récits plutarquéens. Le platonisme est l'obédience philosophique la mieux représentée, à des degrés divers, parmi les convives de Plutarque. À elle se rattache par exemple, tout d'abord, la figure

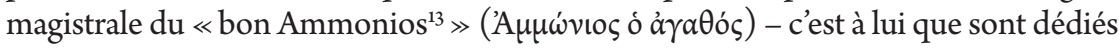
les tout derniers mots des Propos de table -, ce représentant du moyen-platonisme auprès duquel Plutarque s'est formé, durant ses jeunes années athéniennes ${ }^{14}$. Ce sont, également, les jeunes disciples de Plutarque, comme Hagias ou Aristainetos, invités

10 Il s'agit des questions VII, 1 (Contre ceux qui critiquent Platon pour avoir affirmé que la boisson passait dans les poumons), VII, 2 (Qu'est-ce que le «touche-corne » de Platon et pourquoi les graines qui tombent sur les cornes de bouf sont «dures à cuire »?), VIII, 2 (En quel sens Platon a dit que Dieu ne cesse de faire de la géométrie) et IX, 5 (Pourquoi Platon a assigné à l'âme d'Ajax la vingtième place au tirage au sort).

11 Tandis que six questions des Propos de table sont directement tirées des poèmes homériques (II, 5 ; V, 8 ; V, 10; VI, 9 ; IX, 4 ; IX, 13 - le texte de cette dernière question n’a pas été conservé), seules deux autres questions, portant l'une sur l'œuvre de Xénophon (II, 1), l'autre sur les préceptes allégoriques de Pythagore (VIII, 7), se rapportent explicitement à l'étude d'un auteur particulier et de son œuvre.

12 Selon le premier repérage que nous avons effectué pour l'ensemble des neuf livres (hors prologues), le corpus des Propos de table contient plus de 400 références à des auteurs ou à des œuvres du passé grec. Près d'un cinquième d'entre elles se rattachent à l'œuvre d'Homère (le plus souvent à l'Iliade, aux deux tiers). S'agissant de Platon, Angelo Giavatto, dans le précieux relevé qu'il a effectué à la suite de W. C. Helmbold et E. N. O’NeIl (Plutarch's Quotations, Oxford, 1959) pour l'ensemble des Moralia, a recensé 64 références aux dialogues platoniciens dans la totalité des Propos de table (A. Giavatro, « Répertoire des citations de Platon dans les Moralia », dans X. Brouillette, A. Giavatto (éd.), Les dialogues platoniciens chez Plutarque. Stratégies et méthodes exégétiques, Louvain, 2010, p. 131-141; pour une typologie des citations de Platon dans l'œuvre de Plutarque, voir également, dans le même volume, X. Brouilletre, A. Giavatro, « Les dialogues platoniciens chez Plutarque. Une introduction », p. 1-25). Par ailleurs, si les références mobilisées et discutées au cours des échanges touchent à maints domaines de la littérature et des savoirs grecs (épopée, poésie, tragédie, comédie, médecine...), un comptage systématique montre que les traditions platoniciennes sont, pour le champ spécifique de la philosophie, les plus fréquemment abordées, devant les traditions présocratiques (notamment Empédocle, Démocrite et Pythagore) ou péripatéticiennes (Aristote, Théophraste).

13 Plutarque, Propos de table, IX, 15, 748 D.

14 Ammonios apparaît à trois reprises dans le corpus, à chaque fois dans un contexte athénien. Présent parmi les invités du musicien Ératon, en III, 1-2, il est également l'hôte de deux banquets : le premier est raconté en VIII, 3, tandis que le récit du second, réunissant «bon nombre d'érudits » et «à peu

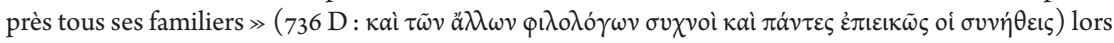
de la fête des Muses, occupe la totalité du livre IX. 
lors d'un banquet chéronéen ${ }^{15}$; le jeune Favorinos d'Arles, présent lors d'un banquet donné par Mestrius Florus dans sa résidence des Thermopyles - mais encore décrit, dans

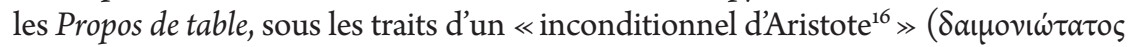

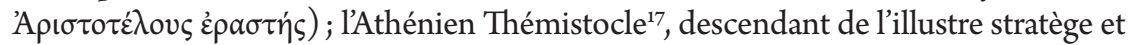
formé lui aussi auprès d'Ammonio ${ }^{18}$, mais rallié au stoïcisme; le grammairien Hylas, enfin, probablement athénien, décrit comme un fin connaisseur de l'œuvre platonicienne ${ }^{19}$.

Le rappel de ces quelques données suffit à entrevoir l'importance de la figure platonicienne dans les Propos de table. Modèle inspirateur de l'écriture plutarquéenne, le philosophe est aussi l'une des références privilégiées de l'univers intellectuel façonné dans la succession des récits de banquets. Pour les convives de Plutarque, cependant, Platon n'est pas seulement le philosophe par excellence, une source de méditation et d'autorité avec laquelle seul Homère, dans le domaine de la poésie, semble pouvoir rivaliser. Certains des épisodes rapportés dans le recueil et les pratiques savantes auxquelles ils sont associés montrent en effet que le commerce avec le philosophe et son œuvre, pour les symposiastes de Plutarque, s'inscrit dans une relation qui dépasse l'admiration ou le simple attachement intellectuel.

\section{Platon, «saint patron » de l'hellénisme et des philosophes}

L'espace ritualisé du banquet, où l'on échange $\left\langle\right.$ mets et mots $^{20} \gg$, est placé, dès le prologue du premier livre ${ }^{21}$, sous le patronage des Muses et de Dionysos. Ce dernier est, en effet, le dieu Lysios ou Lyaios, celui qui délie et libère les brides de la langue ${ }^{22}$. Son pouvoir, conjugué avec l'inspiration que les Muses et les Nymphes concèdent aux sages, aux poètes et autres «maîtres de vérité », fait du banquet une sphère

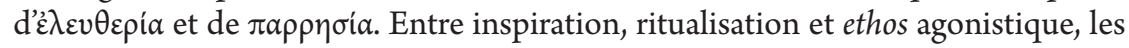
joutes chorales des symposiastes, où chacun se renvoie astucieusement la parole,

15 Au banquet donné à Chéronée (III, 7-9) par Autoboulos, le père de Plutarque, les deux personnages sont désignés $\left(66_{5} \mathrm{~F}\right.$ ) comme ces «garçons qui étudient la philosophie [avec Plutarque] 》 (oi

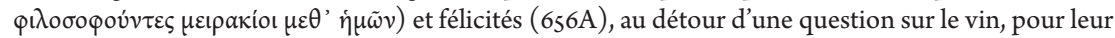

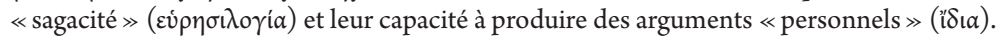

16 Plutarque, Propos de table, VIII, 10, 734 F.

17 Le personnage fait son unique apparition à un banquet donné par Mestrius Florus (I, 9), où la discussion, consacrée aux propriétés nettoyantes de l'eau douce, se porte sur Chrysippe, Aristote et Homère.

18 Plutarque, Thémistocle, 32, 6. Voir sur ce point la notice biographique de PUECH, «Les amis de Plutarque $\gg$, p. 4886.

19 C'est en effet en ces termes, lors de l'unique banquet auquel prend part le grammairien, que le rhéteur Sospis interpelle Hylas au sujet d'un détail dans le récit platonicien du mythe d'Er (IX, 5, 740A) :

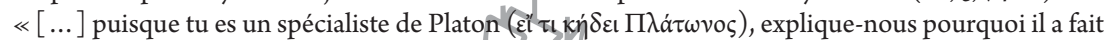
assigner par le sort le vingtième rang à l'âme du fils de Télamon pour venir faire son choix ».

20 Nous nous inspirons de L. Romeri, Philosophesentre mets et mots. Plutarque, Lucien, Athénée autour de la table de Platon, Grenoble, 2002.

21 Plutarque, Propos de table, I (prologue), $612 \mathrm{E}$.

22 Plutarque, Propos de table, I, 2, 613 C. C'est Craton qui s'exprime ainsi, un parent par alliance de Plutarque. Cf. Puech, «Les amis de Plutarque $»$, p. 4843. 
reposent sur la persuasion plutôt que sur la démonstration ; leur but ultime s'exprime en termes d'équilibre, de joie et de plaisir. C'est pourquoi la scène du banquet ne se prête pas à tous les discours, à tous les récits ou à toutes les évocations. Tournant

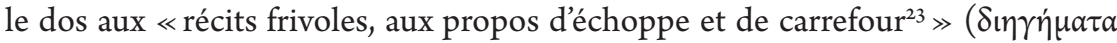

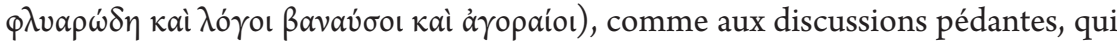
bafouent la dignité de Dionysos, Plutarque recommande de choisir des $\pi \alpha \rho a \delta \varepsilon \dot{\gamma} \gamma \mu \alpha \tau \alpha$ susceptibles d'orienter les participants vers la philosophie et la piété ${ }^{4}$ et de susciter le

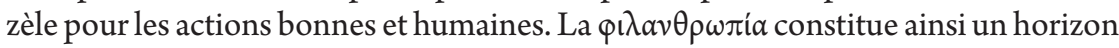
très prégnant de l'univers sympotique ${ }^{25}$. En ces matières, le Platon du Banquet fait évidemment figure de précurseur et de modèle, lui qui, aux dires de Plutarque, évita soigneusement de confondre banquet et palestre, dialogue et lutte ${ }^{26}$. En lieu et place de la puissance musculaire, Platon a eu recours à la souplesse, aux bons exemples et aux récits mythologiques, distillés avec sobriété.

Passé maître dans le maniement des $\pi \alpha \rho \alpha \delta \varepsilon i ́ \gamma \mu \alpha \tau \alpha$ propices à la réussite des banquets, Platon est érigé en modèle dans un double registre : celui des convenances, dont il établit le périmètre, et celui de la postérité des échanges, si tant est que le plaisir des banquets est indissociable de leur pouvoir de remémoration ${ }^{27}$. En effet, la sobriété exemplaire des réunions platoniciennes en garantit l'à $v \alpha$ $\mu \nu \eta \sigma \iota \varsigma$, qui permet de faire revivre les propos tenus et le plaisir qu'ils ont suscité. Ainsi, les discussions de jadis restent fraîches et actuelles, tandis que Socrate et Platon demeurent éternellement vivants, offerts à la jouissance des générations successives, jusquà l'époque de Plutarque et même au-delà ${ }^{28}$. Leur manière de philosopher transcende le temps et l'espace, et fonde les règles fécondes d'un banquet générateur de filiations et de mémoire vivantes. Ainsi, en $698 \mathrm{~F}$, les participants du banquet plutarquéen

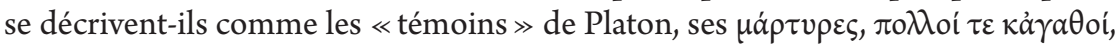
$\ll$ nombreux et de qualité $»$. Le terme de « martyr $\gg$, choisi par Plutarque, renvoie à la sphère juridico-religieuse, puisque les dieux sont fréquemment pris à témoin par les hommes ${ }^{29}$, et oriente, par conséquent, dans le chef de ceux qui se réclament de Platon, vers une affiliation inébranlable, un militantisme engagé en faveur de celui dont la $\delta o ́ \xi \alpha$ et la $\delta \dot{v} \alpha \mu \iota \varsigma$ sont sans pareil ${ }^{30}$.

Pour revivre ou revitaliser le lien entre les participants et leur glorieux ancêtre, le « saint patron » du banquet, quelques rituels sont instaurés, que le texte de Plutarque donne à voir. Ces pratiques construisent la communauté des symposiastes comme le

23 Plutarque, Propos de table, I, 1, 615A.

24 Plutarque, Propos de table, I, 1, 614B.

25 Sur cette valeur, cf. D. Konstan, Friendship in the Classical World, Cambridge, 1997 et surtout J. Ribeiro Ferreira et al. (éd.), Symposion and philanthropia in Plutarch, Coimbra, 2009.

26 Plutarque, Propos de table, I, 1, 614 D-E.

27 Plutarque, Propos de table, VI (prologue), $686 \mathrm{D}:$ : Ils [= Platon et Xénophon] nous ont laissé des modèles à suivre $(\pi \alpha \rho \alpha \delta \varepsilon i \gamma \mu \alpha \tau \alpha)$, non seulement pour ce qui est des réunions et des conversations à table, mais encore de la manière de garder le souvenir $(\mu \varepsilon \mu \nu \tilde{\eta} \sigma \theta \alpha \iota)$ des propos tenus ».

28 Le thème de la réminiscence est déjà au cœur du premier prologue (cf. supra).

29 Voir par exemple A. H. Sommerstein, J. Fletcher (éd.), Horkos. The Oath in Greek Society, Exeter, 2007.

30 Plutarque, Propos de table, VII, 1, $700 \mathrm{~B}$. 
fruit d'une lignée ininterrompue de philosophes descendant de Platon et légitiment leurs discours, à l'ombre du divin ancêtre ${ }^{31}$. Les banquets sont ainsi l'occasion de pratiquer des lectures en commun des textes de Platon ${ }^{32}$ et de faire vivre des communautés herméneutiques, en partie comparables à celles qui émergent, autour des textes sacrés, dans le judaïsme et le christianisme à la même époque ${ }^{33}$. D’ailleurs, même si le débat et la dialectique entre des points de vue différents sont au cœur des pratiques, les groupes réunis autour de Plutarque endossent occasionnellement le rôle d'experts de la parole platonicienne ou de « gardiens du temple », garants d'une certaine forme d'orthodoxie philosophique ${ }^{34}$. Du reste, face à des adaptations des dialogues platoniciens, qu'ils jugent fantaisistes et qui sont alors en vogue à Rome, Plutarque et ses amis expriment une vive indignation en ces termes ${ }^{35}$ :

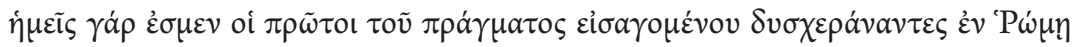

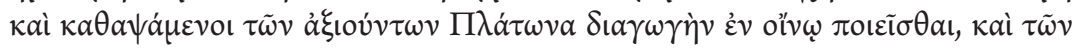

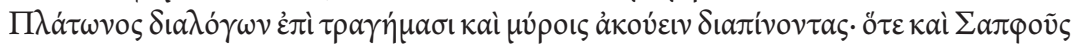

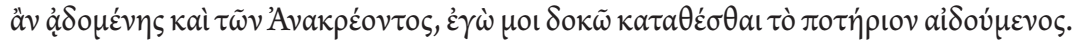

Car nous sommes les premiers à nous être irrités de voir cette pratique introduite à Rome et à nous être attaqués à ceux qui prétendent faire de Platon un passe-temps pour banquets et écouter les dialogues de Platon dans nos beuveries au milieu des friandises et des parfums, alors que, déjà, quand on chante du Sappho ou des vers d'Anacréon, je décide de poser ma coupe en signe de respect.

L’aióś, indiqué ici comme le comportement approprié envers Sappho, Anacréon et a fortiori Platon, est un sentiment complexe, qui renvoie, dans les termes mêmes de Jean Rudhardt, au souci de maintenir pour soi les conditions d'une bonne conscience $^{36}$. Il engage le respect des hiérarchies sociales, la dignité ( $\left.\tau \iota \eta^{\prime}\right)$ que l'on doit aux autres, une retenue prudente, et il traduit en définitive une vénération profonde, parfois craintive en raison du regard quautrui - hommes ou dieux - porte sur le comportement de chacun. La dignité des textes de Platon doit donc être respectée et faire l'objet d'une révérence quasiment « religieuse », qui engage tout à la fois les

31 Sur cette notion de communauté et de lignée, voir KöNIG, Saints and Symposiasts... , p. 40-52 ; K. Eshleman, The Social World of Intellectuals in the Roman Empire: Sophists, Philosophers, and Christians, Cambridge, 2012, p. 177-212.

32 Voir en particulier Plutarque, Propos de table, VII, 2, 700 C. Voir aussi Plutarque, Consolation à Apollonios, $120 \mathrm{D}$, où il annonce qu'il enverra à Apollonios ses réflexions personnelles sur le dialogue

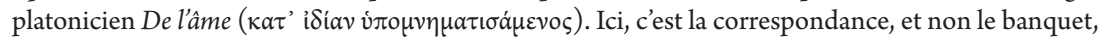
qui sert d'espace au partage herméneutique entre fidèles platoniciens.

33 Sur ces éléments de comparaison et de convergence dans la relation aux « autorités », voir Eshleman, The Social World of Intellectuals ..., p.199-212.

34 En VIII, 2, 718 C, Plutarque suggère, à propos d'une citation que la tradition attribue à Platon : «Je fis observer alors qu'elle ne se trouvait écrite noir sur blanc dans aucun de ses ouvrages, mais qu'elle était suffisamment attestée et de facture platonicienne».

35 Plutarque, Propos de table, VII, 8, 711 D.

36 J. Rudhardt, «Quelques remarques sur la notion d'aidôs », dans É. Delruelle, V. PirenNeDelforge (éd.), Kêpoi : De la religion à la philosophie. Mélanges offerts à André Motte, Liège, 2001, p. 1-21. 
termes mêmes dans lesquels sa pensée s'exprime, mais aussi les gestes qui pourraient les accompagner et les contextes dans lesquels on les convoque.

La manifestation la plus patente de ce que l'on peut raisonnablement considérer comme un culte de Platon apparait dans la première question du livre VIII des Propos de table, intitulée « Sur le jour de naissance de quelques hommes célèbres ; et aussi : sur les prétendues filiations divines ». Plutarque y évoque deux anniversaires célébrés coup sur coup par son cercle d'amis ${ }^{37}$ :

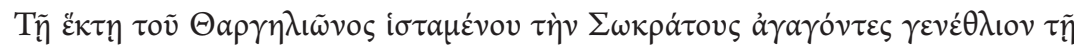

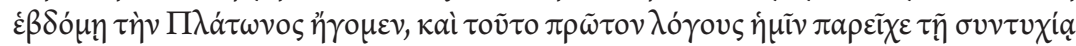

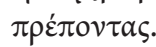

Après avoir célébré le 6 du mois de Thargélion l’anniversaire de Socrate, nous célébrâmes le 7 celui de Platon, ce qui nous fit entamer la conversation sur un sujet approprié à cette coïncidence.

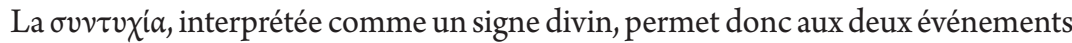

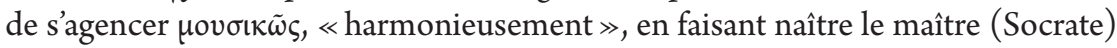
un jour avant son disciple (Platon). Or la date de naissance de Platon autorise un rapprochement ultérieur avec Apollon dont on célèbre, au même moment, à Athènes, la fête des Thargélies, puisque le dieu, lui aussi, serait né le 7 du mois de Thargélion ${ }^{38}$. Nul besoin de s'appesantir sur le symbolisme du chiffre sept, qui évoque la plénitude,

Plutarque, Propos de table, VIII, 1, 717 B.

38 Diogène Laërce (III, 1-2) évoque cette tradition ancienne en ces termes: « Speusippe, dans son ouvrage intitulé Banquet funéraire de Platon, Cléarque, dans son Éloge de Platon, et Anaxilaïde, dans le deuxième livre de son ouvrage Sur les philosophes, rapportent une histoire qui courait à Athènes; Ariston voulut forcer l'hymen de Périctionè, qui était dans la fleur de l'âge, mais il n'y parvint pas ; quand il eut mis un terme à ses tentatives, il vit Apollon lui apparaître. Â partir de ce moment, il s'abstint de consommer son mariage jusquà ce que Périctionè eût accouché. Platon est né, comme le rapporte Apollodore dans sa Chronique, au cours de la quatre-vingt-huitième Olympiade, le septième jour du mois de Thargélion, le jour où les gens de Délos disent qu’est né Apollon » (trad. L. BRIsson, Paris, 1999). Le thème de l'ascendance apollinienne est ensuite repris par Apulée, Platon et sa doctrine, I, 1-2: « Certains prétendent que Platon fut le fruit d'une conception plus auguste encore: une incarnation d'Apollon (Apollonis figuratio) se serait unie à Perictionè. En outre, il naquit dans le mois Thargélion en Attique, le jour où, dit-on, Latone enfanta Apollon et Diane à Délos. La tradition nous apprend que l'anniversaire de la naissance de Socrate tombait la veille [... ] 》 (trad. J. BEAUJEU, CUF, Paris, 1973). Voir aussi l'opinion d'Origène, Contre Celse, I, 37 : «Il s'agit là en réalité de mythes qui ont poussé à imaginer un prodige de ce genre au sujet d'un homme, parce que, jugeait-on, il était d'une sagesse et d'une puissance supérieures à celles de la plupart, il avait reçu de semences supérieures et divines le principe de sa constitution corporelle, comme il convient à ceux qui ont une grandeur plus qu'humaine » (trad. M. BORRET, Cerf, Paris, 1967; voir aussi VI, 8). Enfin, dans les Prolégomènes à la philosophie de Platon, 1, 16-60, on trouve cette jolie formule : «Platon était divin et Apollinien. Qu'il fût divin, c'est ce que révèlent et son propre aveu et certains songes [...]. Ces songes ne sont pas les seuls signes du caractère apollinien de Platon, mais il y a aussi sa manière de vivre, qui était une vie de purification. Car telle est la nature de ce dieu, comme son nom même l'indique. "Apollon" signifie, en effet, "celui qui est séparé de la pluralité", puisque le "a" est un préfixe privatif. En outre, la date de naissance de Platon nous témoigne aussi qu'il était apollinien. Il naquit, en effet, le septième jour du mois de Thargélion, jour où les Déliens célébraient la fête d'Apollon. Or Socrate naquit le sixième jour de ce mois, jour où l'on célébrait l'anniversaire d'Artémis. Ce qui montre bien la primauté de Socrate 
appliqué ici tant aux pouvoirs d'Apollon quà l'enseignement de Platon. On prêtera davantage attention aux propos que prononce Florus en écho au rapprochement calendaire entre Platon et Apollon ${ }^{39}$ :

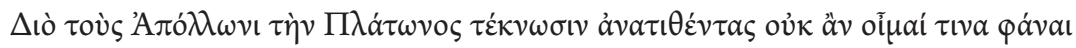

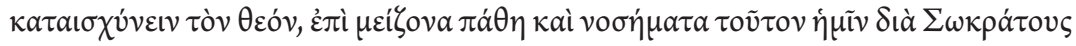

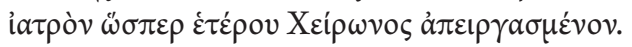

Voilà pourquoi ceux qui font d'Apollon le père de Platon ne sauraient, je pense, être accusés de déshonorer le dieu, qui, en lui, avec l'aide de ce second Chiron que fut Socrate, nous a ménagé un médecin pour des affections et des maladies plus graves que celles du corps.

Plutarque amplifie ensuite ces propos en précisant : «Et en même temps il rappela la vision quaurait eue durant son sommeil le père de Platon, Ariston, et la voix qui lui aurait interdit de s'unir à sa femme et de la toucher pendant dix mois ${ }^{40} \gg$. Ainsi, assimilé à Asclépios, dieu né « miraculeusement » d'un dieu, Platon, le $\theta \varepsilon \tilde{o} о \varsigma \Pi \lambda \alpha \dot{\alpha} \tau \omega \nu$ de la Vie de Périclès, était vénéré comme un médecin de l'âme ${ }^{41}$. C'est pourquoi Tyndare de Lacédémone conclut judicieusement la discussion en acclamant Platon tout en citant Homère ${ }^{42}$ :

"A

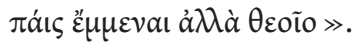

Vraiment il y a bien lieu de chanter et de dire à propos de Platon ces vers : $\ll$ Et il ne semblait pas être fils d'un mortel, mais descendant d'un dieu ».

dans l'ordre du temps comme dans celui de la valeur $\gg$ (trad. J. TrouILlard, CUF, Paris, 1990). Sur la fête des Thargélies et son déroulement à Athènes, voir R. PARker, Polytheism and Society in Ancient Athens, Oxford, 2005, p. 185, 203-204, 481-483.

39 Plutarque, Propos de table, VIII, 1, 717 E.

40 Ibidem.

41 Olympiodore rapporte de la même façon dans sa Vie de Platon, 3: «Quant il mourut, les Athéniens lui donnèrent une sépulture somptueuse et gravèrent sur son tombeau cette inscription : "Apollon engendra deux enfants, Asclépios et Platon, afin que l'un sauve l'âme, l'autre le corps” » (trad. N. D'ANDres et al., 2010).

42 Plutarque, Propos de table, VIII, 1, 717 E. La citation correspond à Iliade, XXIV, 258. Sur les citations homériques chez Plutarque, voir C. BRÉCHET, «L'influence des Alexandrins sur les citations homériques de Plutarque et leur commentaire », dans A. CASANOva (éd.), Plutarco e l'età ellenistica (Atti del convegno internazionale di studi, Firenze, 23-24 settembre 2004), Florence, 2005, p. 243-268; ID., «La lecture plutarquéenne d'Homère : de la Seconde Sophistique à Théodore Métochite », dans La tradition des Moralia de Plutarque de l'Antiquité au début de la Renaissance (Journée d'étude du 30 janvier 2004, Université de Toulouse II-Le Mirail), Pallas, 67, 2005, p. 175-201 ; ID., « Grecs, Macédoniens et Romains au test d'Homère: référence homérique et hellénisme chez Plutarque », dans The Unity of Plutarch's Work: Moralia Themes in the Lives, Features of the Lives in the Moralia (vII Congrès international de l'IPS à Rhethymnon, 4-8 mai 2005), Berlin, 2008, p. 85-109. C. Bréchet note que le jeu des références à l'Iliade et à l'Odyssée, chez Plutarque, sert notamment à associer les Grecs, héritiers des Achéens, et les Romains, descendants des Troyens, suggérant subtilement une communauté de civilisation entre eux, sous l'égide de l'hellénisme, dont Homère est le fondateur et dont les Romains sont les élèves tardifs. 
Le rituel acclamatoire, qui esquisse un hymne théogonique à Platon, est renouvelé à chaque date anniversaire ${ }^{43}$. Cette célébration par les symposiastes de leur saint patron s'accompagnait d'offrandes de mots et trouve son point d'orgue dans une sorte d'épiphanie platonicienne, dans le cadre de la deuxième question du livre VIII, qui fait suite au débat sur la filiation divine de Platon et est intitulée «En quel sens Platon a dit que Dieu ne cesse de faire de la géométrie $\gg$. Un convive lance d'emblée ${ }^{44}$ :

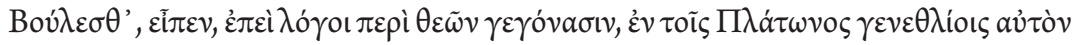

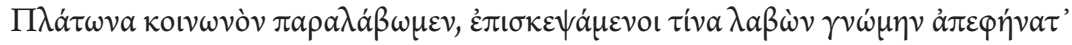

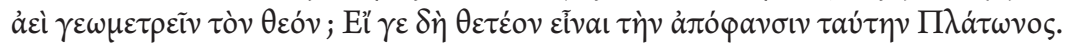

Voulez-vous, puisque la conversation s'est portée sur les dieux, que, à l'anniversaire de Platon, nous fassions participer Platon en personne à notre conversation et examinions en quel sens il a dit que Dieu ne cessait de faire de la géométrie - en admettant qu'il faille bien attribuer cette affirmation à Platon - ?

Par sa pensée, placée $\varepsilon \dot{\zeta} \mu \varepsilon \dot{\sigma} \sigma o v$, dans l'espace de débat et de partage que constitue le banquet, Platon est présent, tel un dieu dont la parole, à l'instar de celle d'Apollon, dont Plutarque fut le desservant à Delphes ${ }^{45}$, est objet d'exégèse par ses prêtres et prophètes. Fils et émule d’Apollon, Platon délivre donc une parole inspirée, divine, volontiers cryptique, que ses héritiers analysent, discutent, transmettent à leur tour dans le cadre du rituel sympotique où Dionysos et Apollon, on le voit, cohabitent harmonieusement. En s’appropriant la pensée du maître par le biais de commentaires

43 La célébration de l'anniversaire de Platon à Athènes est évoquée par Eusèbe, Préparation évangélique, $\mathrm{X}, 3,1-25$ : « Pour fêter l'anniversaire de Platon ( $\tau \grave{\alpha} \Pi \lambda \alpha \tau \omega \dot{\omega} v \varepsilon \mathrm{i} \alpha)$, Longin nous avait invités à Athènes avec plusieurs autres [...]. Prosènès s'exprima à son tour: "Vous avez dépisté les autres plagiaires; mais que ce héros lui-même, Platon, dont nous célébrons aujourd'hui la fête onomastique, a exploité beaucoup de ses prédécesseurs (j’aurais honte [aỉoṽual] de lui appliquer le terme de plagiat), vous ne l'avez plus reconnu" » (trad. G. SCHROEDER, Cerf, Paris, 1991). Voir aussi, sur cet événement, Porphyre, Vie de Plotin, 2: «Aux anniversaires traditionnels de Socrate et de Platon, il sacrifiait et il offrait un repas à ses compagnons; ce jour-là, ceux qui en était capables devaient aussi lire un discours devant l'assemblée »; 15: «J'avais lu, à la fête de Platon, un poème sur le Mariage sacré; et comme une grande partie de ce poème était dite de façon mystique, sous l'effet de l'inspiration, à mots couverts, quelqu'un dit : "Porphyre est fou". Plotin dit, de manière à être entendu de tous : "Tu as montré à la fois le poète, le philosophe et l'hiérophante” » (trad. É. BrÉHIER, revue par S. MORLET, Classiques en poche, Paris, 2013). Voir enfin les Prolégomènes à la philosophie de Platon, 6, 9-22, au sujet d'une femme qui avait consulté l'oracle pour savoir s'il fallait ranger le monument du philosophe parmi les statues des dieux et avait obtenu la réponse suivante : «Tu feras bien d'honorer Platon, le guide d'une sagesse divine. En retour, te viendra la faveur des bienheureux, parmi lesquels cet homme est compté ». On signalera encore, pour terminer, que Laurent de Médicis organisait des fêtes dans sa villa de Careggi le jour de la naissance et de la mort de Platon, le 7 novembre; Marsile Ficin en a conservé le souvenir dans son commentaire de Platon. Il invitait neuf convives : comme les Muses et comme les participants au Banquet de Platon.

44 Plutarque, Propos de table, VIII, 2, 718 C. Il s'agit de Diogenianos de Pergame, un ami de Plutarque auquel il rend hommage aussi dans le De Pythiae oraculis, 395 A. Voir PUECH, « Les amis de Plutarque $\gg$, p. 4846 .

45 Voir J. Boulogne, Plutarque. Un aristocrate grec sous l'occupation romaine, Lille, 1994. 
doxographiques, les participants au banquet légitiment leur propre parole et font rejaillir sur eux la $\delta o ́ \xi \alpha$ et la $\delta \dot{v} v \alpha \mu \varsigma$ de leur divin modèle, tout en reconnaissant néanmoins, avec une modestie toute philosophique, que la vérité est ä $\lambda \eta \pi \tau$

\section{De la mémoire à la présence}

La figure fondatrice et toujours vivante de Platon donne naissance, dans l'espace ritualisé du banquet, à une communauté, au sein de laquelle se transmet un héritage sans cesse réactivé par les pratiques sympotiques. Les performances intellectuelles qui y prennent place, sous forme de questions, citations, évocations, engagent la mémoire culturelle des individus et du groupe et elles façonnent leur identité propre, bien au-delà d'une simple posture mimétique ${ }^{47}$. La citation ou l'évocation des grands poètes ou des philosophes illustres ont donc une vertu psychagogique et

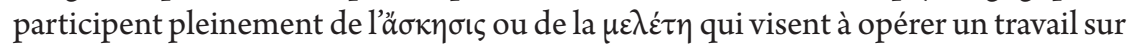
soi et à disposer de formules susceptibles d'être utiles, au jour le jour, comme moyen d'action sur soi-même, comme incitation à faire les bons choix, comme ressource prophylactique ou thérapeutique. En d'autres termes, la convocation des auteurs du passé, de Platon in primis, est une ressource essentielle de la $\pi \alpha$ ¿sía. Lérudition mise en commun et mise en action, comme Plutarque la donne à voir dans les Propos de table, opère en outre à plusieurs niveaux ou échelles.

L’ensemble des banquets regroupés dans les neuf livres, en ce qu'ils mobilisent une petite centaine de savants originaires des quatre coins du monde connu, en dépit d'une forte surreprésentation de la Grèce, en ce qu'ils associent des spécialistes de disciplines très variées et de diverses orientations philosophiques, font écho au cosmopolitisme de l'Empire gréco-romain et au potentiel universel inhérent à l'hellénisme. Le microcosme sympotique se construit au miroir du macrocosme dont Rome se veut le centre et la Grèce le cœur. Les réseaux savants des Propos de table rayonnent largement autour de Plutarque et déploient de proche en proche leurs ramifications dans l'oikoumène, à la faveur de la pax Romana. Cette « étoile » potentiellement en expansion permet aussi de dessiner des cercles concentriques ${ }^{48}$. Ceux-ci se dilatent en effet depuis le cercle familial de Plutarque à Chéronée jusquà la Grèce, pour réunir ensuite la Grèce et Rome,

46 Plutarque, Propos de table, VII, 1, 700 B. Il est question, dans ce passage, d'anatomie humaine et de l'affirmation de Platon, critiquée par certains, que la boisson passe par les poumons.

47 Sur le rôle de la citation dans les échanges intellectuels, qui ne se réduit pas à la simple reproduction d'un modèle, mais constitue une véritable création au départ d'un modèle, voir C. BRÉCHET, « Sur la prétendue dimension mimétique de la citation en Grèce ancienne », dans D. AugER, É. Wolff (éd.), Culture classique et christianisme. Mélanges offerts à Jean Bouffartigue, Paris, 2008, p. 259-273; ID., « Vers une philosophie de la citation poétique : écrit, oral et mémoire chez Plutarque », dans Plutarch's Philosophical Tactics (Dublin, Trinity College, juillet 2005), Hermathena, 182, 2007, p. 101-134.

48 Pour une autre vision concentrique appliquée à Athenes en tant que centre de la Grèce et, au-delà, du monde gréco-romain, voir, à peu près à la même-eque que Plutarque : Aelius Aristide, Panathénaïque, 16: «[...] puisque la Grèce est au centre de la terre entière, l'Attique au centre de la Grèce, la cité au centre de la contrée, et qu'au centre de la cité se trouve son homonyme [= l'Acropole] $\gg($ trad. E. Оudot $)$. 
et rassembler finalement tous les symposiastes dans un vaste espace qui va jusquà la Gaule, l'Asie Mineure et l'Égypte. En dépit de son universalisme affiché, le monde du banquet, avec ses performances intellectuelles, trace une ligne de partage entre les savants et les autres, les dépositaires d'un héritage aussi prestigieux que vivant et le commun des mortels qui n'en a guère connaissance. Bref, c'est l'élite des $\pi \varepsilon \pi \alpha ı \delta \varepsilon v \mu \varepsilon ́ v o l$, promoteurs des valeurs morales dont nous avons parlé ci-dessus, qui se déploie face aux spectateurs invisibles d'une scène savante où ils n'ont pas leur place. Or, comme l'a récemment relevé Jason König ${ }^{49}$, les inscriptions évergétiques qui émanent des cités de l'Empire et qui évoquent le déroulement des grands festivals, souvent à vocation panhellénique, affichent la liste des bienfaiteurs de l'événement et des invités venus des quatre coins du monde, en vertu d'une logique « universalisante » analogue. Il s'agit, dans un cas comme dans l'autre, de mettre en avant le cosmopolitisme et les valeurs partagées, comme l'hospitalité, qui nourrissent le nouvel espace global de l'Empire. L'apport des grandes familles à la construction de cet espace partagé, quoique sélectif, se nourrit significativement de traditions ancestrales qu'il faut faire vivre, à la charnière entre un glorieux passé et un avenir que l'on voudrait radieux.

En définitive, les cercles savants ont à la fois une portée intégrative, au regard du projet d'un Empire multiculturel ayant élu l'hellénisme comme socle et ciment de cette diversité, et une visée élective, celle de donner à voir une élite, investie d'une mission qui s'inscrit plus dans le temps que dans l'espace. Sur ce plan, le cercle est plus judicieusement représenté, dans la littérature sympotique, comme une lignée, une $\delta เ \alpha \delta o \chi \eta$, c'est-à-dire une communauté qui se construit dans la durée et dans la succession, par la transmission d'un héritage ${ }^{50}$. Capable de transcender la contingence historique, la lignée partage à la fois le temps court du banquet, au cours duquel les solidarités sont activées, l'espace d'une performance commune, et le temps long, voire immémorial de la translation du savoir, des filiations et des traditions savantes. $\mathrm{Au}$ croisement du cercle et de la lignée, la communauté plutarquéenne devient une famille, pour utiliser une notion qui dit bien ces deux dimensions de l'expérience sympotique. En faisant de Platon le fils d'Apollon, et de Socrate un second Chiron, en présentant le banquet comme l'assemblée de ses fils spirituels, les amis de Plutarque annoncent la Platonica familia d'Apulée ${ }^{51}$. Ils enracinent leur existence dans un passé mythique, un âge d'or fondateur, porteur de « la joie et la sérénité des choses consacrées, sublimes et célestes $\gg$, que le rituel du banquet sert à réactualiser.

49 KöNIG, Saints and Symposiasts..., p. 81-88. Voir aussi ID., « Self-Promotion and Self-Effacement in Plutarch's Table Talk», dans F. KLotz, K. Oıкоnomopoulou (éd.), The Philosopher's Banquet: Plutarch's Table Talk in the Intellectual Culture of the Roman Empire, Oxford, 2011, p. 195-202. Lanalyse de J. König rejoint ici les remarques formulées par C. Bréchet sur les liens entre oralité, mémoire et statut ontologique de la citation chez Plutarque et les Anciens, comme pratique philosophique et restitution de la parole vive («Vers une philosophie de la citation poétique... », p. 101-105).

50 Sur cette notion, voir en particulier Eshleman, The Social World of Intellectuals..., p. 177-199.

51 Apulée, Apologie, 64: «Nous autres, au contraire, famille de Platon (Platonica familia), nous ne connaissons que la joie et la sérénité des choses consacrées, sublimes et célestes. Que dis-je ? Dans son effort pour s'élever plus haut, cette philosophie a exploré des régions supérieures au ciel même, et s'est arrêtée sur la surface extérieure de l'univers » (trad. P. VALLETTE, CUF, Paris, 1924). 
L'anamnèse joue un rôle crucial dans ce processus de va-et-vient entre le passé et le présent, entre Platon et ses descendants : se remémorer les échanges advenus chez Platon, rejouer les joutes de jadis, convoquer au banc des témoins les grandes figures du passé (les $\pi \alpha \rho \alpha \delta \varepsilon i ́ \gamma \mu \alpha \tau \alpha)$, faire parler les grands auteurs et entretenir avec eux une relation personnelle ${ }^{52}$ - l'un des convives, Philippos de Prousias, affirme ainsi qu'Euripide est son $\varphi$ í $\lambda \circ \varsigma^{53}$ - sont autant de stratégies pour donner corps à une communauté philosophique vivante. La philosophie n'est-elle du reste pas définie comme un «art de vivre ${ }^{54} \gg$, embrassant le passé et le présent dans une même démarche mêlant connaissance et plaisir, savoir et pouvoir ? Comme chez Lucien, dans les Dialogues avec les morts ou dans la Discussion avec Hésiode, le passé est mobilisé comme un horizon accessible, vivifiant, un capital symbolique mis au service du présent, accessible à la jouissance des contemporains. Lorsque, dans le prolongement de l'anniversaire de Platon, les symposiastes proposent, pour le fêter et l'honorer, de travailler sur une de ses pensées - «En quel sens Platon a dit que dieu ne cesse de faire de la géométrie $\gg-$, on invite le glorieux et divin ancêtre à prendre part à

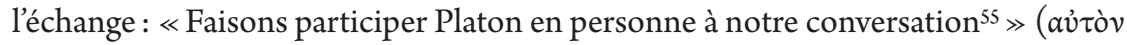

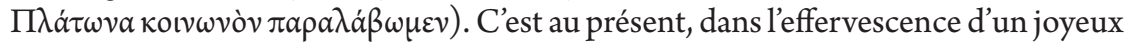
$\sigma v \mu \pi$ ó les auteurs qui incarnent un hellénisme vivant et rayonnant. Jason König a utilement montré, par le truchement d'une analyse du vocabulaire de la citation dans les Propos de table, que le lexique utilisé par Plutarque pour évoquer le recours au témoignage et à l'autorité des Anciens ressortit majoritairement au registre du dialogue et de la communication orale ${ }^{56}$. La mémoire, en somme, se mue en conversation avec les Anciens et en reviviscence d'une présence. La performance collective des symposiastes de Plutarque rejoint ici et fait écho au programme mémoriel des Propos de table, dont Platon, dans le prologue du livre VI, est décrit comme l'inspirateur ${ }^{57}$ :

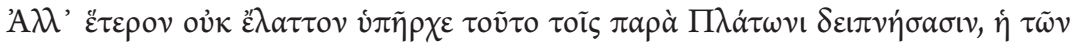

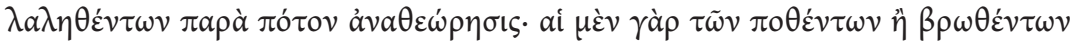

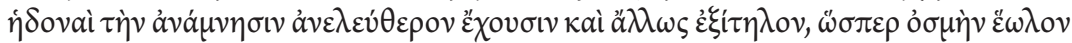

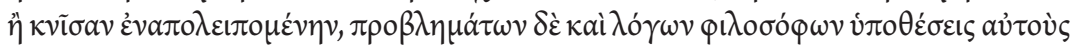

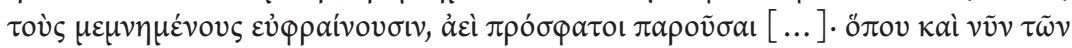

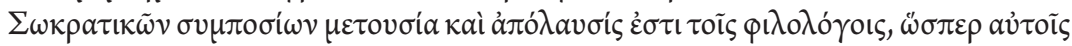

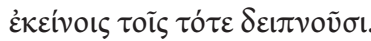

Cependant, un autre avantage non moins précieux était assuré aux hôtes de Platon : la possibilité de se remémorer ce qui s'était dit à table ; car, si le plaisir qu'on a pris à boire et à manger ne laisse qu'un souvenir vulgaire et d'ailleurs fugace, semblable à une senteur qui s'évente ou à des relents de cuisson, les

Cf. supra, n. 42, sur le statut de la citation et son rốte dans l'ethos philosophique.

Plutarque, Propos de table, VII, 7, $710 \mathrm{E}$.

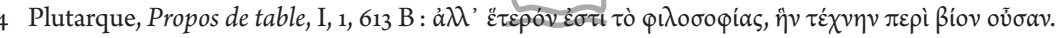

5 Plutarque, Propos de table, VIII, 2, 718 C.

56 KöNIG, Saints and Symposiasts..., p. 76-77.

57 Plutarque, Propos de table, VI (prologue), 686 B-C. 
recherches et les discussions philosophiques restent, toujours fraîches et présentes, des sujets de délectation pour ceux qui s'en souviennent [...]. C'est ainsi que de nos jours encore les lettrés assistent aux banquets de Socrate et en profitent au même titre que ceux qui jadis dînèrent réellement avec lui.

Socrate et Platon, initiateurs du banquet savant dont la qualité garantit la pérennité, constituent bien, pour Plutarque et son petit monde de lettrés gréco-romains, des maîtres à penser et à agir, les passeurs incontournables d'une $\pi \alpha$ เ $\delta$ cí qu 'il faut continuer de transmettre et de faire rayonner par-delà les frontières de la Grèce historique.

\section{Conclusion}

Le traitement et les usages de la figure platonicienne dans les Propos de table permettent de ressaisir, à partir d'un cas singulier, les opérations et les dynamiques que mobilise, dans le contexte de l'Empire gréco-romain, la construction d'une mémoire savante partagée. Comme premier des philosophes « par la réputation et l'autorités ${ }^{8} \gg$, Platon est élevé, dans les conversations et les pratiques qui composent l'univers plutarquéen des banquets de lettrés, à la dignité d'un « saint patron », d'une figure tutélaire au contact de laquelle se constitue une part de l'identité des symposiastes. Placée à l'articulation de la communauté herméneutique et de la communauté rituelle que forment les convives de Plutarque, la référence platonicienne opère, pourrait-on dire, comme un $\sigma \dot{v} \mu \beta o \lambda o v$, marquant successivement l'appartenance à un cercle et le rattachement à une tradition. Garantie de légitimation et d'intégration, l’adhésion à la figure « mythifiée $\gg$ de Platon, telle qu'elle s'actualise dans le temps et la performance collective du banquet, acquiert aussi une dimension mobilisatrice où la science, dans l'exploration de la mémoire culturelle grecque et par l'émulation avec les Anciens, se joue non seulement comme partage ritualisé, mais aussi comme instance morale et discours de vérité.

Le thème plutarquéen du divin Platon apporte ainsi un éclairage singulier sur les recompositions qui accompagnent, à l'orée du siècle des Antonins, l'épanouissement d'un Empire gréco-romain. Forgée à partir de matériaux en circulation dès le $\mathrm{IV}^{\mathrm{e}}$ siècle av. J.-C. parmi les disciples de l'Académie, cette tradition semble acquérir dans les Propos de table une signification et une portée nouvelles. Nous avons vu par exemple le succès que le motif de l'ascendance apollinienne de Platon, que l'on célébrait encore au $\mathrm{III}^{\mathrm{e}}$ siècle à Athènes ou à Rome lors de réunions comparables à celles qu'évoque Plutarque, devait rencontrer auprès des $\pi \varepsilon \pi \alpha \iota \delta \varepsilon v \mu \varepsilon ́ v o$ de tout l'Empire, de la Platonica familia d'Apulée aux cercles néo-platoniciens réunis autour de Plotin ou de Longin. La figure plutarquéenne du divin Platon, reprise, amplifiée par la littérature doxographique, tendait ainsi à devenir l'expression d'un hellénisme renouvelé, conçu comme le langage et le référent d'un espace culturel désormais étendu aux dimensions de la Méditerranée. 


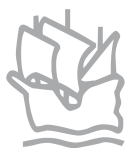

(C) BREPOLS PUBLISHERS

THIS DOCUMENT MAY BE PRINTED FOR PRIVATE USE ONLY

IT MAY NOT BE DISTRIBUTED WITHOUT PERMISSION OF THE PUBLISHER. 